\title{
Hierarchical Shape Statistical Model for Segmentation of Lung Fields in Chest Radiographs
}

\author{
Yonghong Shi ${ }^{1}$ and Dinggang Shen ${ }^{2, *}$ \\ ${ }^{1}$ Digital Medical Research Center, Fudan University, Shanghai, 200032, China \\ Yonghong. Shi@fudan. edu.cn \\ ${ }^{2}$ Department of Radiology and Biomedical Research Imaging Center \\ University of North Carolina, Chapel Hill, NC 27599 \\ dgshen@med.unc. edu
}

\begin{abstract}
The standard Active Shape Model (ASM) generally uses a whole population to train a single PCA-based shape model for segmentation of all testing samples. Since some testing samples can be similar to only sub-population of training samples, it will be more effective if particular shape statistics extracted from the respective sub-population can be used for guiding image segmentation. Accordingly, we design a set of hierarchical shape statistical models, including a whole-population shape model and a series of sub-population models. The whole-population shape model is used to guide the initial segmentation of the testing sample, and the initial segmentation result is then used to select a suitable sub-population shape model according to the shape similarity between the testing sample and each sub-population. By using the selected subpopulation shape model, the segmentation result can be further refined. To achieve this segmentation process, several particular steps are designed next. First, all linearly aligned samples in the whole population are used to generate a whole-population shape model. Second, an affinity propagation method is used to cluster all linearly aligned samples into several clusters, to determine the samples belonging to the same sub-populations. Third, the original samples of each sub-population are linearly aligned to their own mean shape, and the respective sub-population shape model is built using the newly aligned samples in this sub-population. By using all these three steps, we can generate hierarchical shape statistical models to guide image segmentation. Experimental results show that the proposed method can significantly improve the segmentation performance, compared to conventional ASM.
\end{abstract}

Keywords: Active shape model, Hierarchical shape statistics; Chest radiograph.

\section{Introduction}

The utility of image processing technique in diagnostic chest radiology has increased with the growing acceptance of digital radiography. Many methods, such as automatic

Corresponding author. This work was partially supported by Shanghai Leading Academic Discipline Project (Project No. B112) and Science and Technology Commission of Shanghai Municipality (Project No. 06dz22103). 
detection of lung nodules, characterization of interstitial disease, and delineation of ribs, have been developed. In all these applications, the information inside the lung region is most interesting. Thus, the segmentation of lung regions becomes an important image processing procedure that has to be performed in most practical applications [1-3].

Various methods have been applied to segment the lung fields from posterioranterior chest radiographs [1-5], and they roughly fall into four categories: 1) rulebased segmentation methods have been used to detect the outline of ribcage or the diaphragm; 2) pixel-based methods were proposed to classify each pixel of an image into either lung field or background based on a multi-scale filter bank of Gaussian derivatives and a K-NN classifier; 3) hybrid methods were formulated by combining rule-based methods and pixel-based classification for lung field segmentation; and 4) deformable model-based methods, such as active shape model and active appearance model have been successfully applied in lung field segmentation.

Among these four categories of segmentation algorithms, the active shape model (ASM) developed by Cootes et al. [4] was a prosperous starting point because of its ability to incorporate a priori information extracted from a training set and its flexibility to represent object shapes. In ASM, the use of PCA-based shape statistics trained on population samples ensures that the segmentation can produce plausible shapes. However, current shape-based segmentation methods generally use the whole population samples to train a single PCA-based shape model and use it for segmentation of all testing samples. Since some testing samples can be similar to only sub-population of training samples, it will be more effective if particular shape statistics extracted from the respective sub-population can be used for guiding the segmentation [10-14]. However, it is not pre-known which sub-population shape information should be used for a new test image before segmenting it.

We accordingly design a set of hierarchical shape statistical models, including a whole-population shape model and a series of sub-population shape models, to hierarchically guide image segmentation. In particular, the whole-population shape model is used to guide the initial segmentation of the testing sample, and the resulted segmentation is used to select a suitable sub-population shape model according to the shape similarity between the testing sample and each sub-population. Thus, by using the selected sub-population shape model, the segmentation result can be further refined.

To achieve this designed segmentation process, we will produce a set of hierarchical shape statistical models in the training stage as follows. First, all linearly aligned samples in the whole population are used to generate a whole-population shape model. Second, an affinity propagation method [9] is used to cluster all linearly aligned samples into several clusters, to determine samples belonging to the same sub-populations. Third, the original samples of each sub-population are linearly aligned to their own mean shape, and the respective sub-population shape model is built using the newly aligned samples in the sub-population. By using all these three steps, we can generate hierarchical shape statistical models to guide image segmentation.

This paper is organized as follows. Section 2 introduces a strategy of our hierarchical shape statistical models for segmenting lung fields from chest radiographs. Section 3 describes the experiments, and Section 4 concludes the paper. 


\section{Method}

\subsection{Summary of ASM Algorithm}

For better describing our proposed method, we first summarize the main idea of ASM algorithm, and point out its potential limitation [2,4]. The ASM scheme consists of two main elements: a global shape model and a local appearance model.

A global shape model is built from a set of training samples, e.g., segmented lung fields $[2,6]$. Each training image is described by $n$ correspondence points, using a shape vector $\mathrm{x}=\left(x_{1}, y_{1}, \ldots, x_{n}, y_{n}\right)^{\mathrm{T}}$. All shape vectors are linearly aligned by minimizing the sum of squared distances among all aligned shape vectors. By calculating the mean shape $\bar{x}$ and the covariance matrix, the principal modes can be estimated. Thus, a new shape $\mathrm{x}$ can now be represented as $\mathrm{x}=\overline{\mathrm{x}}+\Phi \mathrm{b}_{\mathrm{x}}$, where $\Phi$ contains the principal modes of variation of the shape model and $b_{x}$ contains shape parameters. It has been proved that, if the specific shape statistics can be used for guiding the segmentation, the accuracy of segmentation can be highly improved [5]. This motivates us to build hierarchical shape statistical models for hierarchically guiding the image segmentation in this study.

In ASM, a local appearance model is constructed for each landmark by using the normalized first derivative profile. For enhancing the performance of ASM, several improvements for the local appearance model have been proposed. For example, the moments of local histograms extracted from the filtered versions of the images using a filter bank of Gaussian derivatives are considered to enhance the accuracy of ASM [7]. Also, scale invariant feature transform (SIFT) [8] is used to significantly improve the performance of ASM [5]. In this study, we will use SIFT to construct local appearance model.

\subsection{Hierarchical Shape Statistical Models}

In Introduction, we mentioned that we will build a set of hierarchical shape statistical models to guide image segmentation. A whole-population shape statistical model will be first built from all linearly aligned samples (c.f. Fig. 1(F)) as described in Section 2.1 above. Then, a series of sub-population shape statistical models will be built to better represent the shape variations within each sub-population. Subpopulations will be generated from all aligned samples using an affinity propagation method [9], which can adaptively cluster the samples into a number of clusters (or sub-populations). The number of clusters (or sub-populations) will be automatically determined, based on the given shape similarity measure. Once we know the samples belonging to a particular sub-population, we can obtain their original shapes and then linearly normalize them into their own mean shape (c.f. Fig. 1(A) (E)), which can be very different from the mean shape of whole population (c.f. Fig. 1(F)). Accordingly, we can use those particularly aligned samples to build a sub-population shape statistical model for each sub-population, using PCA technique. Details of this process are described next. 


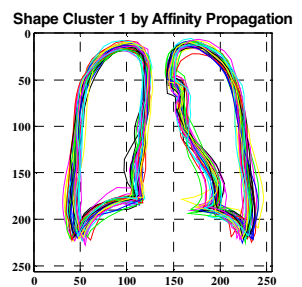

A

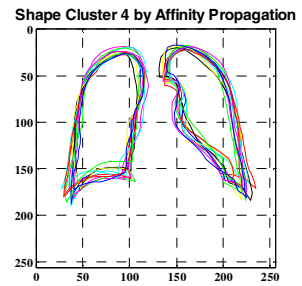

$\mathrm{D}$

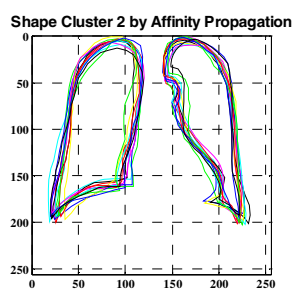

B

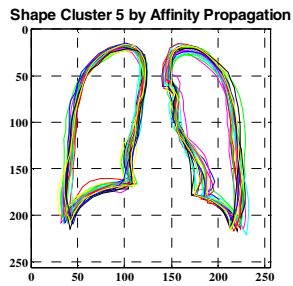

$\mathrm{E}$

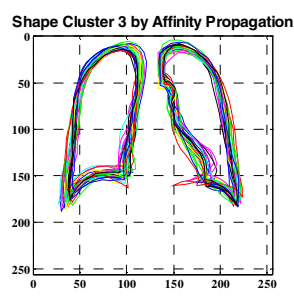

$\mathrm{C}$

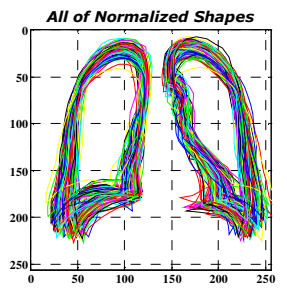

$\mathrm{F}$

Fig. 1. Normalized samples in whole population (F), or in sub-populations (A E). Subpopulations are clustered by affinity propagation. There are totally five sub-populations from our normalized training samples. Note, the original samples belonging to the same subpopulation are re-normalized to their own mean shape $(\mathrm{A} \sim \mathrm{E})$, to represent their own variations.

\section{Clustering of sub-populations}

Clustering data by identifying a subset of representative examples (or exemplars) is important in many applications including ours. The $k$-means algorithm can be used to find such "exemplars", by first randomly choosing an initial subset of data points and then iteratively refining it. However, this works well only when initial choice is close to a good solution. Contrary to the $k$-means algorithm, affinity propagation method [9] simultaneously considers all data points as potential exemplars, and then recursively exchanges real-valued messages between data points until a high-quality set of exemplars and corresponding clusters emerges. Affinity propagation method can find clusters with much lower error than other methods, and it can complete in less time.

Thus, we select this method to cluster our training samples into a number of subpopulations. For example, for those spatially normalized samples (c.f. Fig. 1(F)), we obtained five sub-populations using affinity propagation (c.f. Fig. 1(A) (E)). Original samples in each of these five sub-populations are re-normalized to their own mean shape. By overlapping all re-normalized shapes in each sub-population, we can obtain five figures in Fig. 1(A E), which is different from the overlay of all normalized shapes in the whole population Fig. 1(F).

These five figures clearly indicate that five sub-populations have very different emphases. For example, a sub-population in Fig. 1(A) has shapes of larger size, compared to other sub-populations. For the sub-populations in Fig. 1(B) and Fig. 1(D), the size of right lung (left in the figure) is both larger than that of left lung, while the shapes of left lung in these two sub-populations are very different. On the other hand, sub-populations in Fig. 1(C) and Fig. 1 (E) have different sizes of lung, although their 


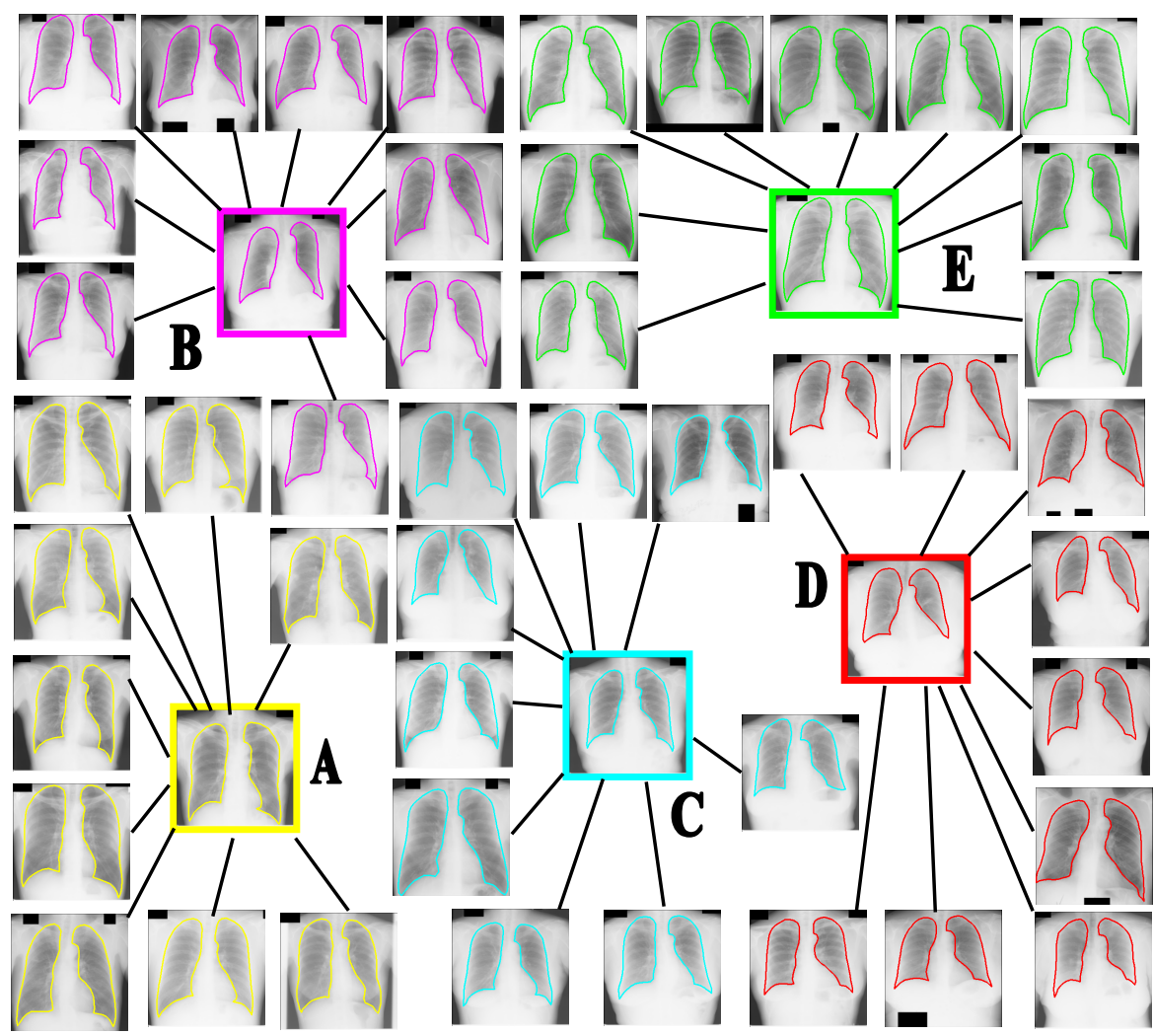

Fig. 2. Original shape samples in five sub-populations of chest radiographs. Exemplars are highlighted by colored boxes. Shapes in the same sub-population are drawn by same color.

overall global shapes are similar. This example intuitively indicates the importance of using sub-population shape statistics for better guiding image segmentation.

Fig. 2 shows the selected original samples in each subpopulation. It can be observed that different sub-populations have very different original shapes.

\section{Construction of hierarchical shape statistical models}

Both whole population shape statistical model and a series of sub-population shape statistical models are built using their own samples, as detailed below:

- The whole population shape statistical model is built from all samples. All samples will be first normalized to the common shape space, to remove the difference of scale, size, shape and position among samples. The normalized samples are shown in Fig. 1(F) for visual inspection. Then, using PCA, these normalized samples can be used to build a whole-population shape statistical model, as used in ASM.

- These normalized samples are clustered into sub-populations, such as five subpopulations in our study (c.f. Fig. 1(A E) and Fig. 2), by the affinity propagation method. Then, all original samples belonging to the same sub-population are re-normalized to their own mean shape (c.f. Fig. 1(A E)). Thus, using PCA, a 
sub-population shape statistical model can be built for each sub-population using its own normalized shape samples.

\subsection{Summary of Our Algorithm}

Our algorithm has two components, i.e., training and testing. In the training stage, we build hierarchical shape statistical models, which include a whole-population shape statistical model and a series of sub-population shape statistical models, as detailed in the subsection above.

In the testing stage, for a given new testing sample, we first use SIFT-based local appearance model to deform our whole-population shape model. The obtained segmentation will be constrained by the whole-population shape statistics. After the segmentation is converged, we use the segmented shape to compute its similarity to each of sub-populations. The most similar one is selected as the particular sub-population that the testing sample belongs to. Thus, we can use the corresponding sub-population shape statistical model to refine the segmentation of the testing sample until the segmentation procedure converges.

\section{Experiments}

The performance of our algorithm is evaluated by a JSRT/SCR database [2, 6]. The 247 cases in this database are subdivided in two folds. Each fold contains an equal amount of normal cases and abnormal cases. Images in one fold were segmented with the images in the other fold as training set, and vice versa. All of the original radiographs were down-sampled to the 256 by 256 resolution images. Two quantitative measures are used to evaluate the performance of the algorithms, i.e., the average overlay percentage and the average contour distance between automated segmentation result and manual segmentation result.

To evaluate the performance of our hierarchical shape statistical model (SIFT-H), we compare the following four methods. SIFT-H denotes our algorithm which uses SIFT features for lung field matching and hierarchical shape statistical models for shape constraining. Intensity- $H$ denotes the method that is similar to SIFT- $H$, except we use image intensity and gradient features to replace the SIFT features for lung field matching. The last two methods, SIFT-W and Intensity- $W$, are, respectively, similar to SIFT-H and Intensity-H, except that SIFT-W and Intensity-W use only whole-population shape statistics for shape constraining.

Some qualitative segmentation results by SIFT-H are provided in Fig. 3. The top row shows the manual segmentations, and the bottom row shows the results by SIFT$H$. It can be seen that the results by SIFT-H are similar to the manual segmentations.

For quantitative comparison, the average overlay percentage and the average contour distance between the segmentation results and the ground truth of all 247 images are reported in Table 1 and Table 2, respectively. It can be seen that, when hierarchical shape statistical models are used, the average overlay percentages of SIFT-H $(93.6 \%)$ and Intensity- $H(89.1 \%)$ are much higher than those of SIFT-W $(92.0 \%)$ and Intensity-W(87.0\%). Similar conclusion can be drawn for the average contour distances in Table 2. It can be observed that the average contour distance is decreased 

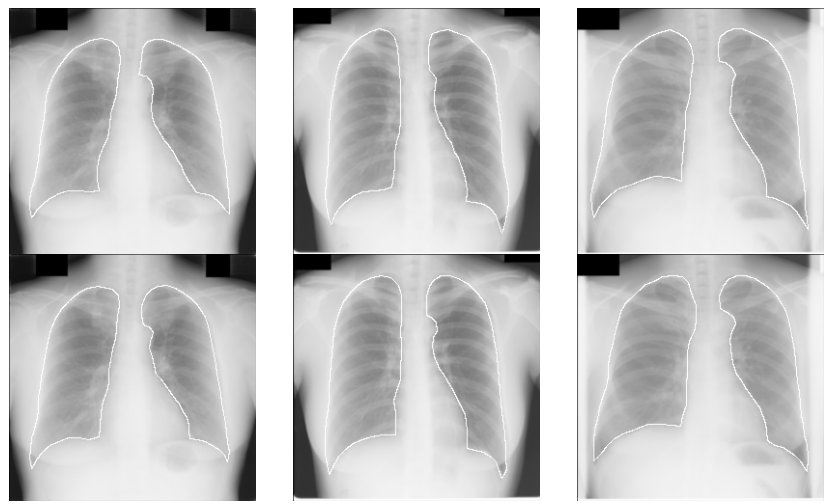

Fig. 3. Three chest radiographs with (top) manual and (bottom) automated segmentation of lung fields by SIFT- $H$

Table 1. Average overlay percentage between manual segmentation and automated segmentation of all 247 images in the JSRT/SCR database (\%)

\begin{tabular}{ccccc}
\hline Algorithm & Mean \pm std & Minimum & Median & Maximum \\
\hline SIFT $-H$ & $93.6 \pm 2.88$ & 80.0 & 93.9 & 96.3 \\
Intensity $-H$ & $89.1 \pm 4.42$ & 70.6 & 90.8 & 95.8 \\
SIF - - $W$ & $92.0 \pm 3.1$ & 78.3 & 92.8 & 96.1 \\
Intensity $-W$ & $87.0 \pm 7.4$ & 60.8 & 89.2 & 95.4 \\
\hline
\end{tabular}

Table 2. Average contour distance between manual segmentation and automated segmentation of all 247 images in the JSRT/SCR database (unit in pixel)

\begin{tabular}{ccccc}
\hline Algorithm & Mean \pm std & Minimum & Median & Maximum \\
\hline SIFT $-H$ & $1.56 \pm 0.62$ & 0.85 & 1.53 & 4.62 \\
Intensity $-H$ & $2.82 \pm 0.77$ & 0.94 & 2.76 & 5.63 \\
SIFT $-W$ & $1.78 \pm 0.78$ & 0.96 & 1.64 & 6.79 \\
Intensity $-W$ & $3.10 \pm 1.95$ & 1.03 & 2.36 & 9.69 \\
\hline
\end{tabular}

from SIFT-W (1.78 pixels) and Intensity-W (3.10 pixels) to SIFT-H (1.56 pixels) and Intensity- $H$ (2.82 pixels), respectively, because of using hierarchical shape statistics. All of these results indicate that, no matter which kind of image features are used, the methods using hierarchical shape statistical models outperform those using only a whole-population shape statistical model.

\section{Conclusion}

We have presented a hierarchical shape statistical model to hierarchically guide the segmentation of lung fields from chest radiographs. This hierarchical shape statistical model includes both whole-population shape statistics and sub-population shape statistics. The clustering of sub-populations from the whole training samples is completed 
by an affinity propagation technique. Then, samples belonging to the same subpopulation are used to train the sub-population shape statistical model using PCA technique. In the applications, the whole-population shape statistical model is first used to guide the initial segmentation of a new test sample. The resulted segmentation is then used to determine which sub-population shape statistics should be used to refine the segmentation of the test sample. By using this proposed hierarchical segmentation strategy, the segmentation performance is highly improved, compared to the ASM using only whole-population shape statistics and the ASM using intensity/gradient features, rather than SIFT features. In this paper, the two-level shape statistical models are used, which can be potentially extended to multiple levels.

\section{References}

1. van Ginneken, B., ter Haar Romeny, B.M., Viergever, M.A.: Computer-Aided Diagnosis in Chest Radiography: a Survey. IEEE Trans. on Medical Imaging 20(12), 1228-1241 (2001)

2. van Ginneken, B., Stegmann, M.B., Loog, M.: Segmentation of Anatomical Structures in Chest Radiographs using Supervised methods: a Comparative Study on a Public Database. Medical Image Analysis 10, 19-40 (2006)

3. Luo, H., Gaborski, R., Acharya, R.: Automatic segmentation of lung regions in chest radiographs: a model guided approach. In: ICIP 2000, vol. 2, pp. 483-486 (2000)

4. Cootes, T.F., Taylor, C.J.: Statistical Models of appearance for Computer Vision. Technical Report, Wolfson Image Analysis Unit, University of Manchester (2001)

5. Shi, Y., Qi, F., Xue, Z., Chen, L., Ito, K., Matsuo, H., Shen, D.: Segmenting Lung Fields in Serial Chest Radiographs Using Both Population-based and Patient-specific Shape Statistics. IEEE Trans. on Medical Imaging 27(4), 481-494 (2008)

6. Shiraishi, J., et al.: Development of a Digital Image Database for Chest Radiographs with and without a Lung Nodule: Receiver Operation Characteristic Analysis of Radiologists' Detection of Pulmonary Nodules. American Journal of Roentgenology 174(1), 71-74 (2000)

7. van Ginneken, B., Frangi, A.F., Staal, J.J., ter Haar Romeny, B.M., Viergever, M.A.: Active shape model segmentation with optimal features. IEEE Trans. on Medical Imaging 21(8), 924-933 (2002)

8. Lowe, D.G.: Distinctive Image Features from Scale-Invariant Keypoints. International Journal of Computer Vision 60(2), 91-110 (2004)

9. Brendan, J.: Frey and Delbert Dueck. Clustering by Passing Messages between Data Points. Science 315, 972-976 (2007)

10. Heap, A.J., Hogg, D.C.: Improving specificity in PDMs using a hierarchical approach. In: British Machine Vision Conference 1997, Colchester, Essex (1997)

11. Bregler, C., Omohundro, S.: Surface learning with applications to lipreading. In: Advances in neural information processing systems, vol. 6 (1994)

12. Stegmann, M.B., Larsson, H.B.W.: Motion-compensation of cardiac perfusion MRI using a statistical texture ensemble. In: Magnin, I.E., Montagnat, J., Clarysse, P., Nenonen, J., Katila, T. (eds.) FIMH 2003. LNCS, vol. 2674, pp. 151-161. Springer, Heidelberg (2003)

13. Stegmann, M.B., Larsson, H.B.W.: Fast registration of cardiac perfusion MRI. In: Proceeding of International Society of Magnetic Resonance in Medicine, Toronto, Canada (2003)

14. Cootes, T.F., Taylor, C.J.: A mixture model for representing shape variation. Image and Vision Computing 17(8), 567-573 (1999) 